\title{
Merck blocks 'safer' gene therapy trials
}

Washington

Gene therapy researchers at the Baylor College of Medicine in Houston, Texas, who recently reported success with the 'gutless' adenoviral vector in animals may not get to test the vector in humans.

The pharmaceutical company, Merck, which owns the licence on a technique used to produce the vector, is refusing to extend its material transfer agreement (MTA) with Baylor, saying that it does not want to be liable for any problems the vector might cause in clinical trials.

Conventional adenoviral vectors usually have only one or two small regions deleted, but 'gutless' (also known as 'fully deleted' or 'helper dependent') adenoviral vectors have a large chunk of their viral genome excised. This means that they could be used to avoid two problems that have limited the usefulness of other adenoviral vectors: inflammation and lack of long-term expression.

Viral genomes in adenoviral vectors trigger immune responses, including inflammation. This is thought to have played a role in the death of 18-year-old Jesse Gelsinger, the first death directly attributed to gene therapy (see Nature 401, 517; 1999). In a later hearing, Merck's former senior vice-president Thomas Caskey testified that the 'gutless' vectors might provide a safer alternative to earlier generations of adenoviral vectors.

Animal experiments reported by Arthur Beaudet, chairman of molecular and human genetics at Baylor, at a conference in Colorado last month, reinforce that view, says Beaudet. In these experiments, Lawrence Chan, professor of molecular and cellular biology and medicine at Baylor, tested the vector in mouse models for inherited high cholesterol.

The experiments resulted in gene expression and lowered plasma cholesterol levels for at least six months. Whereas controls treated with a conventional adenoviral vector had marked inflammation in the liver, says Chan, mice that received the 'gutless' adenoviral vector experienced no significant inflammatory response.

After the experiments but before Gelsinger's death, Beaudet asked Merck to extend the MTA for a tool used to manufacture the 'gutless' vectors. The tool removes a large chunk of the vector's genome, yet still allows it to replicate. Merck denied the request, citing its concerns about liability.

The tool in question emerged from efforts in the early 1990s by a number of scientists - including Caskey, who was then at Baylor — to develop 'gutless' viruses that needed a second, 'helper' virus to replicate. A research group headed by Frank Graham, professor of biology and pathology at McMaster University in Canada, subsequently solved the problem of eliminating the helper virus in the final vector preparations by excising the viral packaging signal.

Merck, which bought a licence on Graham's patent, retains intellectual property rights on it. Even if Baylor researchers sign a liability waiver, the US Food and Drug Administration could still hold the company to "ultimate regulatory responsibility", says Larry Hirsch, vice-president of public affairs at Merck Research Laboratories in Whitehouse Station, New Jersey.

Merck's refusal to extend the Baylor MTA for the vector-producing tool beyond preclinical use is "not unique to gene therapy", says Hirsch. "It's the way we handle all products in early stages of development." He adds that the vector is "nowhere near" ready for testing in a clinical trial.

Chan says the refusal is "frustrating". The Baylor team's only alternative, beyond abandoning the 'gutless' adenoviral vector, is finding a new way to create one. While that remains a possibility, it would probably require years of work. Producing large enough quantities of the vector for humans remains a problem, adds Graham. Meanwhile, Merck plans to continue developing and testing the vector on its own. Paul Smaglik

\section{Celera's shotgun approach puts Drosophila in the bag}

\section{Washington}

Celera Genomics this week announced that it has sequenced the genome of the fruitfly Drosophila melanogaster. The announcement was made at the annual meeting of the American Association for the Advancement of Science, and the sequence will be published next month in the journal Science.

Celera researchers claim that their success in sequencing such a large, complex eukaryotic genome is a vindication of their whole-genome - or shotgun - approach to sequencing (see Nature 401, 729; 1999). Gene Myers, head of Celera's computational biology group, says they met their goals simply on the basis of assembling the sequence data.

These goals included sequencing at least 95 per cent of the euchromatin (that part of the genome containing the vast majority of genes), meeting standards for accuracy and completeness accepted by the National Institutes of Health for human sequencing, and having no gap larger than 150,000 base pairs and an error rate of less than 1 in 10,000 .

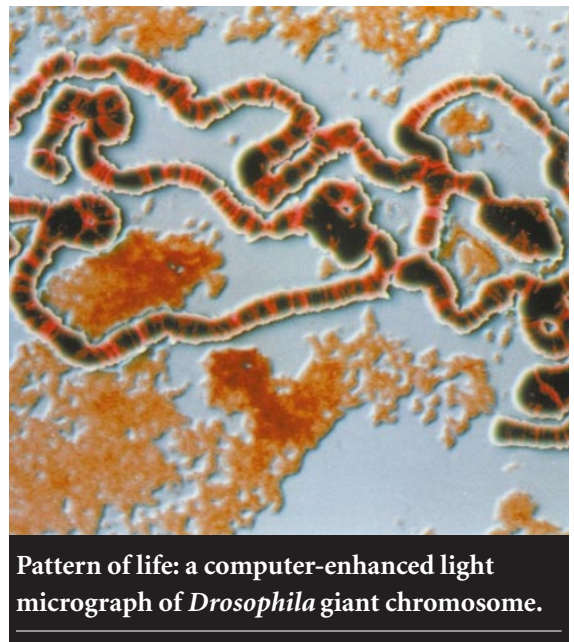

In the programme abstract for the meeting, Myers wrote that preliminary trials suggest that 99.97 per cent or more of the euchromatic portion of the genome will be assembled.

Celera collected more than 3 million shotgun paired reads from the Drosophila genome over a four-month period ending last September. Of the 180 million base-pair
(Mbp) genome, $120 \mathrm{Mbp}$ was sequenceable DNA. Work began last November to close the 1,600 gaps in the sequence. These made up 2.65 Mbp of gaps straight out of the assembler. Their efforts may well "continue for another year or more," says Adams.

Celera say that most of these gaps are places where they have no data, rather than gaps caused by repeat sequences. It has been estimated that there are roughly 13,601 genes in the genome, although this number is expected to "evolve" over the months ahead.

Myers estimates that, from Celera's experience with Drosophila, the problem in obtaining the human sequence will be the larger scale of the genome rather than the assembly of sequence data.

In the fly, an assembler computes overlaps between reads in less than 13 hours on a four-processor platform. Myers says that by arranging assembly to be concurrent with data collection, Celera's assembler should achieve a comparable result in the human genome with a three-month computation, and on a tenprocessor platform. Natasha Loder 